Documentation et bibliothèques

DOCUMENTATION BIBLIOTHËQUES

\title{
Priorités du gouvernement et participation des milieux professionnels au développement d'une politique d'information documentaire
}

\section{Monique Potin}

Volume 25, numéro 3, septembre 1979

URI : https://id.erudit.org/iderudit/1054319ar

DOI : https://doi.org/10.7202/1054319ar

Aller au sommaire du numéro

Éditeur(s)

Association pour l'avancement des sciences et des techniques de la documentation (ASTED)

ISSN

0315-2340 (imprimé)

2291-8949 (numérique)

Découvrir la revue

Citer cet article

Potin, M. (1979). Priorités du gouvernement et participation des milieux professionnels au développement d'une politique d'information documentaire. Documentation et bibliothèques, 25(3), 117-123.

https://doi.org/10.7202/1054319ar
Résumé de l'article

L'auteur examine attentivement les dernières réalisations du gouvernement québécois en matière d'information documentaire et fait le bilan des propositions incluses dans le livre blanc La politique québécoise du développement culturel et le livre vert Pour une politique québécoise de la recherche scientifique. Elle démontre la nécessité pour les spécialistes de la documentation, devant le succès mitigé de plusieurs de leurs interventions, d'être de plus en plus agressifs et convaincants.
Tous droits réservés (c) Association pour l'avancement des sciences et des techniques de la documentation (ASTED), 1979
Ce document est protégé par la loi sur le droit d'auteur. L'utilisation des services d'Érudit (y compris la reproduction) est assujettie à sa politique d'utilisation que vous pouvez consulter en ligne.

https://apropos.erudit.org/fr/usagers/politique-dutilisation/ 


\title{
Priorités du gouvernement et participation des milieux professionnels au développement d'une politique d'information documentaire
}

\author{
Monique Potin
}

Service pédagogique

Université de Montréal

L'auteur examine attentivement les dernières réalisations du gouvernement québécois en matière d'information documentaire et fait le bilan des propositions incluses dans le livre blanc La politique québécoise du développement culturel et le livre vert Pour une politique québécoise de la recherche scientifique. Elle démontre la nécessité pour les spécialistes de la documentation, devant le succès mitigé de plusieurs de leurs interventions, d'être de plus en plus agressifs et convaincants.

The author examines very carefully the latest achievements of the Quebec government in the field of documentary information and evaluates the propositions included in the white paper La politique québécoise du développement culturel and the green paper Pour une politique québécoise de la recherche scientifique. She demonstrates the necessity for documentation specialists to become more and more agressive and convincing following the mitigated success of several of their interventions.

El autor examina atentamente las ultimas realizaciones del gobierno de Quebec relativas a la información documental y resume las proposiciones contenidas en el « libro blanco » titulado La politique québécoise du développement culturel $y$ en el «libro verde» titulado Pour une politique québécoise de la recherche scientifique. Demuestra que es preciso que, frente al éxito mitigado de varias intervenciones suyas, los especialistas de la documentacion se muestren más agresivos y convincentes.

La scène bibliothéconomique québécoise a été traversée, depuis le début de 1978, par toute une série d'événements à forte saveur politique. Se sont succédées, entre autres, en 1978: une Table ronde sur la planification des services documentaires québécois en avril, la parution du livre blanc La politique québécoise du développement culture/ ${ }^{1}$ en juin, la Conférence socio-économique sur les industries culturelles en décembre; et, en mars 1979, la diffusion du livre vert Pour une politique québécoise de la recherche scientifique ${ }^{2}$.

1. Québec, Ministère d'État au Développement culturel, La politique québécoise du développement culturel,.Québec, Editeur officiel, 1978, 2 vol., $472 p$.

2. Québec, Ministère d'État au Développement culturel, Pour une politique québécoise de la recherche scientifique, Québec, Éditeur officiel, 1979, 222p. 


\section{Importance des événements politiques}

Plusieurs caractéristiques de ces quatre événements en dénotent l'importance, aussi bien au niveau du gouvernement et des groupes intéressés que du grand public.

\section{Implication directe des ministres}

Les ministres O'Neill (Communications) et Laurin (Développement culturel) participaient à la Table ronde et à la Conférence socio-économique; le ministre Vaugeois (Affaires culturelles) assistait lui aussi à la Conférencon socio-économique; les deux livres sont signés par le ministre Laurin.

\section{Instauration de mécanismes de consultation par le gouvernement}

En l'espace d'une année, le gouvernement québécois a amorcé un processus fort complexe de consultation et d'information auprès des groupes d'intérêt et du grand public. Les rencontres avec les groupements se sont multipliées, à leur demande ou à l'initiative des ministres. Les politiques énoncées dans les deux livres font l'objet d'intenses consultations, formelles ou informelles, et suscitent de nombreux mémoires ${ }^{3}$.

\section{Le caractère public des événements}

Les deux livres ont fait l'objet d'une très large diffusion auprès du grand public et des groupes d'intérêt. La Conférence socio-économique a reçu de la part des média d'information un traitement aussi important que n'importe quelle conférence

3. Un mémoire conjoint a été déposé en décembre 1978 à la Conférence socio-économique par l'Association des bibliothécaires du Québec ( $A B Q)$, l'ASTED et la Corporation des bibliothécaires professionnels du Québec (CBPQ), intitulé: Participation des bibliothèques au développement culturel. La CBPQ soumettait en février 1979 au ministre Laurin le document Pour des québécois mieux informés dans un Québec en développement. résumé dans l'article d'Alain Perrier "La Corporation et le développement culturel; l'an l', Argus, vol. 8, no 2 (mars-avril 1979), 30-31. économique gouvernementale sur un sujet plus traditionnel que les industries culturelles.

\section{La portée et la nouveauté des sujets abordés}

Le fait d'insérer la consultation sur la politique culturelle dans un cycle de conférences socio-économiques sectorielles donne une importance nouvelle à un secteur que l'on a trop tendance à marginaliser et donc à minimiser; la dimension économique, et non plus seulement politique, des industries culturelles est ainsi mise en valeur.

La politique du livre, établie en 1971 , est remise sur la table pour révision.

Des thèmes comme l'établissement d'une planification nationale des services documentaires et le développement de la recherche au Québec sont pris au sérieux par le gouvernement provincial; ils font l'objet de propositions fermes et concrètes à inclure dans leur développement organisé.

Bref, les spécialistes de l'information documentaire, individuellement et en groupe, ont l'occasion de connaître les intentions gouvernementales; ils ont "voix au chapitre" dans l'établissement des priorités par les dirigeants politiques, dans les secteurs qui touchent de près le monde de la documentation: la vie culturelle, la recherche scientifique et technique, la planification d'un système documentaire québécois.

\section{La Table ronde sur la planification des services documentaires québécois, avril 1978}

Cette Table ronde réunissait des représentants des ministères des Communications (dont le ministre lui-même), des Affaires culturelles et de l'Éducation, de la Bibliothèque nationale du Québec, des associations professionnelles, le ministre d'État au Développement culturel Camille Laurin et trois experts étrangers. 
Elle vient couronner une série d'efforts entrepris par des membres de la profession et par l'ASTED: une rencontre fermée sur la bibliothéconomie québécoise en janvier 1975 et les deux congrès annuels de 1976 et de 1977, axés sur le thème de la coordination des services documentaires québécois.

Au cours de cette Table ronde, le ministre Laurin annonce la parution de deux livres, l'un sur la politique du développement culturel, l'autre sur la politique de la recherche scientifique; il affirme que des mesures concrètes sont prévues être adoptées dans un délai de deux ans. II dévoile aussi le projet d'un «Institut national de planification" ayant un rôle d'étude, de planification, de concertation et d'incitation en regard d'un système national d'information documentaire.

L'ASTED, dans un rapport déposé à cette Table ronde ${ }^{4}$, fait les recommandations suivantes:

- la reprise, par l'ouverture de trois postes, du programme de catalogage avant publication à la $\mathrm{Bi}$ bliothèque nationale du Québec ${ }^{5}$;

- l'établissement d'un réseau pleinement québécois de télécatalogage coopératif en mode interactif6;

- la création d'un Conseil des services d'information documentaire au Québec qui assurerait la coordination, tant au niveau régional que sectoriel, d'un réseau national des services documentaires. II aurait des pouvoirs de planification, d'orientation et d'intervention, et relèverait du Premier Ministre ${ }^{7}$.

\section{Le livre blanc La politique québécoise du développement culturel et ses suites}

Le livre blanc, diffusé en juin 1978, annonce une politique d'ensemble articu-

4. "Les services d'information documentaire au Québec: bilan et propositions». Montréal, ASTED, $1978,52 p$.

5. Ibid, p. 45.

6. Ibid, p. 46-48.

7. Ibid, p. 49-51. lée autour du comité ministériel permanent du développement culturel, comité dirigé par le ministre d'État Camille Laurin ${ }^{8}$. II prévoit la création imminente d'un Institut québécois de recherche sur la culture ${ }^{9}$; cet Institut s'occuperait autant de recherche fondamentale, à long terme, sur la nature même de la culture québécoise, que de recherche orientée et appliquée. II fournirait des statistiques menant à l'établissement de "comptes culturels". Enfin cet Institut de recherche jouerait un rôle de coordination, d'incitation et de soutien au niveau de la documentation sur la culture québécoise.

La politique du livre de 1971 serait “améliorée et renforcée» ${ }^{10}$. Cette politique impose actuellement aux institutions subventionnées de s'approvisionner en livres et manuels scolaires exclusivement auprès des libraires agréés. Les mesures de renforcement indiquées sont principalement:

- l'élargissement du système d'agrément aux éditeurs et aux distributeurs qui sont propriété québécoise à cent pour cent;

- la révision du système de tabelle (taux de change des monnaies étrangères, pratiqués par les distributeurs) et de remise;

- le renforcement des contrôles afin d'assurer le respect de cette politique du livre.

Si cette politique du livre «musclée» ne produisait pas les résultats escomptés, une Centrale du livre pourrait être créée, pour distribuer efficacement livres et périodiques en langue française ${ }^{11}$.

Les publications gouvernementales devraient jouir d'une meilleure diffusion grâce à diverses mesures financières et organisationnelles incitant les libraires à s'y intéresser et améliorant les services de l'Éditeur officiel' ${ }^{12}$.

8. Québec, Ministère d'État au Développement culturel, La politique québécoise..., p. 138-140.

9. Ibid, p. 140-143.

10. Ibid, p. 340-341.

11. Ibid, p. 342.

12. Ibid, p. 349; il faut mentionner en particulier une politique de dépôt pour les librairies et les bibliothèques. 
Enfin une politique de la lecture est établie; elle est axée sur le développement des bibliothèques publiques et bibliothèques centrales de prêt (BCP), et sur une campagne publicitaire systématique d'incitation à la lecture ${ }^{13}$; une nouvelle bibliothèque centrale de prêt a été inaugurée à Rivière-du-Loup en mars 1979 et une dixième BCP est prévue sur la Côte Nord ${ }^{\mathbf{1 4}}$; des programmes d'aide financière sont aussi prévus pour soutenir les efforts des municipalités et des BCP existantes.

Tous ces projets ont été repris et précisés à l'occasion de la Conférence socio-économique sur les industries culturelles dans un document de travail préparé par le Secrétariat des conférences $^{15}$.

La politique du livre a même fait l'objet d'un projet de loi, dévoilé à la mi-janvier 1979 par le quotidien Le Devoir.

Les réactions à ces diverses propositions eurent libre cours lors des rencontres organisées par les ministères, par le biais de mémoires et dans les journaux. Le mémoire commun des trois associations en bibliothéconomie rappelle que «le québécois est un sous-lisant, avant d'être un sous-lisant de lecture québécoise." ${ }^{16}$ Le mémoire met surtout en lumière que la politique du livre constitue une aberration économique, qui "produit des résultats contraires à ceux espérés. "17 A défaut de l'éliminer, le mémoire suggère des modifications précises en vue d'améliorer cette politique: l'établissement d'un double réseau de librairies, le renforcement des normes d'agrément dans le sens de la qualité du service, l'énumération des catégories d'ouvrages à exclure de la loi, la modification du système de tabelle. Le Devoir jugeait "Carrément inacceptable» ${ }^{18}$ le système de contrôle et de surveillance

13. Ibid, p. 392-393.

14. Discours du ministre Vaugeois cité dans les Nouvelles de l'ASTED, no 119 (mars-avril 1979), 28

15. Les industries culturelles; hypothèses de développement, Québec, Secrétariat des conférences socio-économiques, 1978, 100p.

16. Participation des bibliothèques..., p.7.

17. Ibid, p. 10

18. Éditorial de Lise Bissonnette, «Entre Soutien et Carcan», Le Devoir, 30 janvier 1979. de l'application de la loi de la politique du livre, tel que prévu dans le projet de loi ${ }^{19}$.

Tant que la loi ne sera pas passée, les jeux ne seront pas faits et les spécialistes en information documentaire ne seront pas assurés que les intérêts premiers de leurs clientèles sont pris en compte. Cependant, avec des représentants qui ont dans leurs serviettes des propositions concrètes d'amélioration, le monde de la documentation ne peut être mieux placé pour exercer une influence sur la législation future, en matière de politique du livre.

\section{La politique de recherche}

Le livre vert sur la recherche scientifique constitue le deuxième volet de l'effort gouvernemental annoncé par le ministre Laurin. Le thème de la recherche concerne la bibliothéconomie à double titre: en tant que secteur de recherche à développer, et en tant que support indispensable à la recherche. La parution du livre vert, en mars 1979, a été suivie presque immédiatement d'actions de consultation et d'information de la part du ministère, avec sollicitation de mémoires.

Eric de Grolier a tenté d'établir un portrait de la recherche bibliothéconomique au Québec ${ }^{20}$. Ses résultats rejoignent le tableau général de la recherche tracé dans le livre vert: retard de la recherche québécoise sur le reste du Canada; absence de coordination et de leadership de la part des gouvernements québécois; cloisonnement et manque d'articulation des diverses activités de recherche; manque de connaissance de l'état de la recherche; manque de diffusion des travaux effectués. II serait intéressant de compléter le travail d'Éric de Grolier en reprenant le cadre fourni par le livre vert, qui divise la recherche en trois secteurs: universitaire, gouvernementa! et industriel. Le livre vert répond à la principale recommandation d'Éric de Grolier: il annonce la volonté

19. «Politique du livre; rencontre avec le ministre des Affaires culturelles", Nouvelles de l'ASTED. no 118 (janvier 1979), 6.

20. «Pour un programme de recherche orientée en science de l'information au Québec». Argus, vol. 8, no 2 (mars-avril 1979), 18-22. 
arrêtée du gouvernement d'intervenir dans l'établissement d'une politique de la recherche en vue d'établir un plan d'orientation et des priorités propres au Québec. Le livre vert propose la création de structures ministérielles, consultatives et administratives destinées à asseoir cette politique de la recherche encore inexistante ${ }^{21}$.

L'Institut québécois de recherche sur la culture semble tout désigné pour soutenir la recherche en information documentaire, laquelle n'a pu s'institutionaliser encore en milieu gouvernemental ou universitaire. Son statut d'institut public et la volonté de l'organiser en fonction des problèmes, plutôt que par disciplines ${ }^{22}$, semblent des dispositions particulièrement bien adaptées au champ interdisciplinaire des sciences de l'information. Les deux autres axes que le gouvernement désire privilégier sont: la formation du personnel de recherche et la diffusion de l'information scientifique et technique. Là aussi, nous rejoignons le diagnostic d'Éric de Grolier sur la recherche en science de l'information: la priorité devrait être accordée à un inventaire des sources d'information au Québec (comprenant la main-d'oeuvre et l'analyse des besoins) et à l'établissement de «systèmes et réseaux régionaux d'information". Concernant l'information documentaire en tant que support à la recherche, le livre vert prône ${ }^{23}$ :

- la création d'un réseau québécois de documentation et de diffusion;

- la création d'un organisme consacré au développement et à la coordination des services d'information scientifique et technique (I.S.T.);

- l'accélération et l'amélioration de la formation des spécialistes en information scientifique et technique;

- la promotion de l'information scientifique et technique de langue française;

- des activités de sensibilisation des utilisateurs à l'importance de I'I.S.T., en particulier du patronat et des syndicats;

21. Québec, Ministère d'État du Développement culturel, Pour une politique québécoise..., p. 177186

22. Ibid p. 187-190.

23. Ibid, p. 195-204
- la modification de la loi de la Fonction publique et des habitudes administratives qui bloquent actuellement la divulgation de l'information de nature scientifique élaborée au sein de la fonction publique;

- la création d'un organisme chargé de favoriser la vulgarisation scientifique, avec pour point d'appui l'association des communicateurs scientifiques.

Le livre vert accorde, dans ses conclusions, une place non négligeable à l'information documentaire, en lui consacrant un des quatre paragraphes du sixième et dernier chapitre «Mesures et dispositifs d'ensemble». Cependant, certaines remarques doivent tempérer l'impression que l'information documentaire reçoit enfin, de la part des gouvernants, la place et la priorité qui lui reviennent:

- le paragraphe spécifique s'étend sur dix pages d'un ouvrage de plus de deux cents pages. Hors de ces dix pages, la dimension documentaire apparaît très peu dans le reste du document. Sauf erreúr, il faut lire jusqu'à la page 119 (où le terme «bibliothèques" apparaît pour la première et dernière fois) avant de trouver quelque mention de l'information documentaire; l'Institut canadien de l'information scientifique et technique est cité une fois (page 157); le terme «documentation scientifique et technique» apparaît une fois (page 169); la dimension d'information du CRIQ apparaît une fois (page 166). La recherche gouvernementale ne semble pas évaluer l'importance de l'information documentaire, puisqu'il n'en est jamais question dans le chapitre qui est consacré à ce secteur ${ }^{24}$. On a l'impression que les préoccupations majeures manifestées dans le paragraphe du sixième chapitre deviennent secondaires, sinon inexistantes, lorsqu'on examine en détail chaque secteur de la recherche scientifique longuement décrit dans le livre vert; 
- les mesures préconisées dans les dix pages ${ }^{25}$ semblent vouloir rester pour la plupart au niveau des désirs et des voeux. On cherche vainement un engagement ferme du gouvernement de prendre l'initiative de faire avancer les dossiers. Tous les obstacles et difficultés sont énumérés; mais on reste sur sa faim, avec pour toutes perspectives un vaste panorama de suggestions d'action que devraient entreprendre les milieux professionnels.

Nous aurions aimé trouver des phrases commençant par exemple comme ceci: un groupe de travail s'est attelé à la tâche de donner vie à... Nous aimerions que Le Devoir découvre un avant-projet de loi établissant un organisme "musclé» de coordination des services d'I.S.T.

Bref, les démarches et explications provenant des milieux de l'information documentaire se retrouvent en filigrane dans dix pages du livre vert mais ne semblent pas avoir été intégrées dans la conception de la politique de la recherche et dans les mesures concrètes qui en découlent. Ils nous revient de continuer, sans relâche, les actions d'information et de pression auprès des pouvoirs publics, afin que ceux-ci intègrent véritablement l'information documentaire à leurs priorités de développement.

\section{Conclusion}

Le gouvernement québécois s'est engagé dans le développement d'une politique culturelle globale, et il entend agir d'ici la fin de 1980. Cette politique culturelle intéresse les spécialistes de l'information documentaire au plus haut point. Ceux-ci ne peuvent se permettre de rester en marge des actions entreprises; ils doivent s'imposer comme partenaires à part entière dans le choix des priorités et dans leur concrétisation. Parmi les priorités avancées par le gouvernement, rappelons-en certaines:

25. Ibid, p. 194-204.
- la création d'un Institut québécois de recherche sur la culture;

- la formation du personnel de recherche;

- la diffusion de l'information scientifique et technique avec création d'un réseau québécois et d'un organisme de coordination;

- l'amélioration de la politique du livre ${ }^{26}$;

- la modification de la loi de la Fonction publique;

- des activités de sensibilisation auprès du patronat et des syndicats.

Le gouvernement a accordé la priorité au développement des bibliothèques publiques et à une campagne d'incitation à la lecture. Mais il reste encore beaucoup à faire dans le secteur de l'enseignement primaire et secondaire; on ne voit pas comment le québécois deviendra un «lisant", si les structures scolaires ne favorisent pas le développement des habitudes de lecture ${ }^{27}$.

Les spécialistes de l'information documentaire doivent développer des mécanismes de concertation et d'intervention auprès des pouvoirs publics, en vue de continuer de représenter avec succès les intérêts de leurs usagers.

Non seulement le monde de la documentation doit-il se concerter, mais il doit aussi s'appuyer sur d'autres organismes populaires et locaux qui constituent les intermédiaires indispensables en vue de faciliter la démocratisation de la culture. Jacques Depaigne fait de la participation des associations et mouvements populaires une des clés de la véritable démocratie culturelle $^{28}$; il définit la démocratie cultu-

26. Au moment où nous rédigeons la conclusion de cet article, le gouvernement vient de publier le projet de loi 51 relatif à la politique du livre.

27. Dans une conférence de presse, lundi le 18 juin 1979, le ministre Vaugeois annonce la publication, à l'automne 1979, d'un plan conjoint de développement des bibliothèques scolaires.

28. Jacques Depaigne, Les politiques culturelles en Europe, Strasbourg, Conseil de l'Europe, 1978, $64 p$. 
relle par le fait de favoriser l'épanouissement de la créativité de chacun, de susciter un comportement de producteur plutôt que de consommateur. N'est-ce pas là l'occasion pour les spécialistes de l'information documentaire de repenser et d'élargir leur rôle culturel?

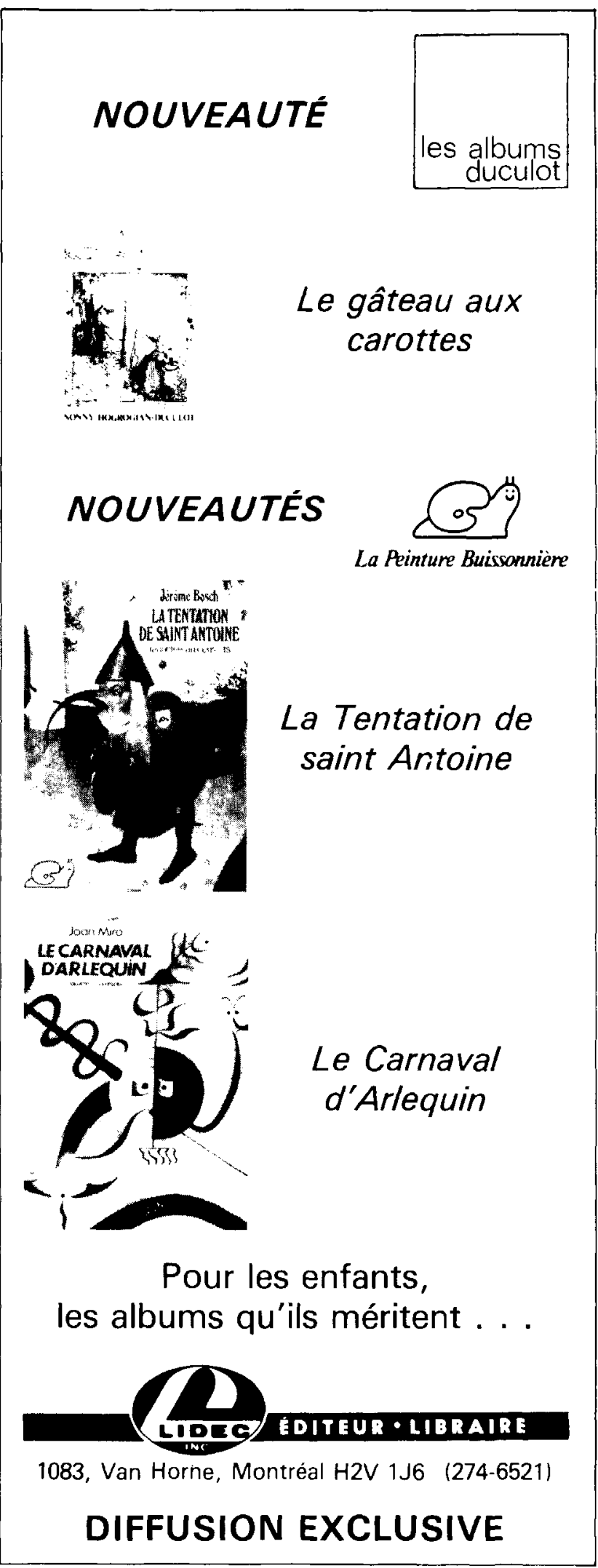

